The same upward trend was found with regard to disease duration before arthroplasty: a significant difference between group A and group $B$ (16.98 y vs 21.66 y; $p=0.03$ ) and between group $A$ and group $C(16.98$ y vs $22.93 y ; p=0.00)$. The rate of implant survival at 5, 10 and 15 years were comparable (from $84 \%$ to $89 \%$ ); whereas $50 \%$ of eligible implants lasted 20 years or more (Figure 2).

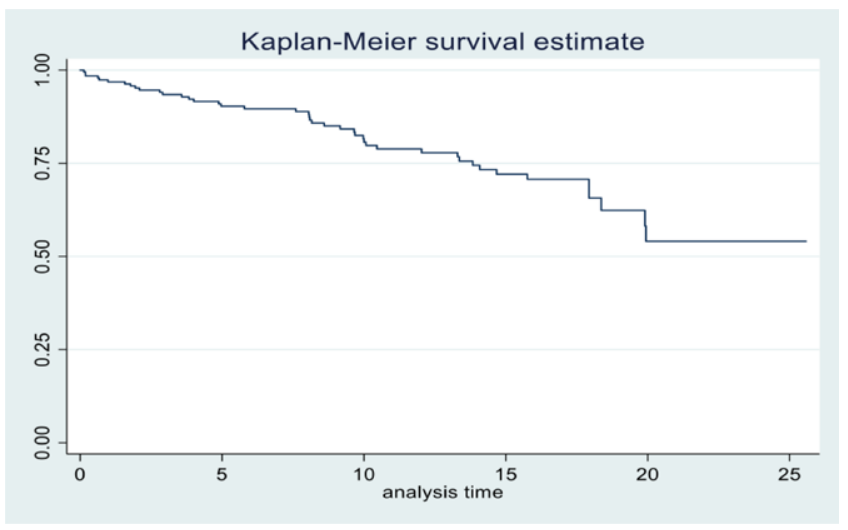

Figure 2. Kaplan Meier survival curve of implants.

The year of surgery was found to be significantly related to implant survival [Hazard Ratio (HR) 1.001, confidence interval (Cl) 1.0001-1.0006; $p<0.001$ ] as well as the presence of complications (HR 3.69, $\mathrm{Cl} 1.82-7.48 ; \mathrm{p}<0.001$ ) in multivariate analysis. Furthermore, prostheses of polyarticular RF-neg patients had more possibilities to last longer than those of S-JIA patients (HR $0.23, \mathrm{Cl}$ $0.09-0.53 ; p=0.00$ ) as well as implants of all polyarticular JIA (RF-pos and neg together $)(p<0.001)$

Conclusion: We observed an upward trend of both age at arthroplasty and disease duration before the first arthroplasty over time. JIA category, year of implants and the presence of complications significantly affected implant survival. Future researches should assess functional outcome and survival of implants according to medical therapy and different surgical approaches.

Disclosure of Interests: None declared

DOI: 10.1136/annrheumdis-2020-eular.1747

\section{THU0505 MUSCULOSKELETAL ULTRASOUND MONITORING DURING MTX TAPERING IN JIA: A PROSPECTIVE BLINDED COHORT STUDY}

F. Licciardi ${ }^{1}$, M. Dellepiane ${ }^{1}$, C. Covizzi ${ }^{1}$, F. Figus ${ }^{2}$, I. Azzolin², D. Montin ${ }^{1}$, A. lagnocco ${ }^{2} .{ }^{1}$ Regina Margherita Children Hospital, Rheumatology Department, Turin, Italy; ${ }^{2}$ Università degli Studi di Torino, Turin, Italy

Background: Juvenile idiopathic arthritis (JIA) is the most common chronic rheumatic disease in children. Musculoskeletal ultrasound (MSUS) is a reliable tool in the assessment of chronic inflammatory arthropathies. MSUS in JIA has demonstrated a higher sensitivity for detecting synovitis and tenosynovitis as compared to physical examination. The occurrence of subclinical synovitis (Sub$\mathrm{S}$ : MSUS+/ physical examination -) seems more frequent in wrist and foot joints; the clinical significance of Sub-S in real-life practice is still debated. Methotrexate (MTX) is the most widely used first-line DMARD in JIA therapy. Weekly treatment with MTX leads to clinical remission (CR) in $50-70 \%$ of patients. After a variable period of CR (usually 6-18 months), MTX is discontinued. Relapse rate after MTX suspension ranges between $40-50 \%$; no predictors of disease flare have been identified so far.

Objectives: We designed a cohort study in order to explore if MSUS monitoring during MTX tapering was able to predict disease flare.

Methods: JIA patients in CR (as defined by the JADAS score) for at least 12 months were enrolled in the study. Patients at first attempt of suspension (G1) were tapered as follows: 1 week of suspension every 3 weeks for 3 months +1 dose every 2 weeks for 3 months; if CR persisted, MTX was stopped. Patients who had a previous flare during/after MTX tapering (G2) had a similar tapering schedule but the step with 1 MTX dose every 2 weeks lasted 6 months. All patients underwent a complete MSUS of 48 joints every 3 months; clinicians who performed physical examinations and follow-up were blinded to US findings for the entire study period.

Results: 18 consecutive patients were enrolled between April 2018 and September 2019; patients had prevalently oligoJIA (55.5\%) and RF- polyJIA (22.2\%). Patients had been treated with MTX for 24.7 months (17.7-48.3), CR had been achieved 4.2 months after MTX start; $61.1 \%$ were at their first attempt of MTX tapering (G1). Baseline MSUS: at TO MSUS detected 9/18 patients (50.0\%) with Sub-S (MSUS+). Affected sites at T0 were distributed as follows:
4 MCP joints, 9 MTP joints, 1 f-IP joints, 11 knees. No significant differences resulted in comparing demographic and baseline disease features between MSUS- and MSUS+ patients at TO. Follow-up MSUS: 14 patients (77.8\%) completed the entire study protocol, 4 patients are still ongoing. 7 patients relapsed: $42.9 \%$ during tapering, 1 of them relapsed during a VZV infection and was excluded from further analysis. We considered as $\mathrm{T}_{\text {last }}-\mathrm{MSUS}$ the last available MSUS before relapse or final MSUS (i.e. three months after MTX withdrawal) for not-relapsed subjects.

At $T_{\text {last }} 8$ patients had at least 1 Sub-S. Sub-S per patient at $T_{\text {last }}$ were more than Sub-S at T0 (2.85 vs $0.53 p=0.03)$ but the presence of Sub-S was not related with disease flare (50.0 vs $44.4 \% \mathrm{p}=1$ ). MSUS found 27 Sub-S of the small joints (sMSUS): $88.9 \%$ were in the feet, they had an OMERACT grading of 1. sMSUS+ patients were older ( 8.7 vs $3.9, p=0.002)$ therefore a weight-induced sub-S not related with JIA could be presumed.

Kaplan-Meier curves were analyzed comparing MSUS results at T0 and $T_{\text {last }}$ both considering all Sub-S and excluding small feet joints (pMSUS). The best performance was achieved with MSUS at $T_{\text {last }}$ and pMSUS (figure below, $\mathrm{p}=0.11)$.

\section{Remission proportions: last pMSUS}

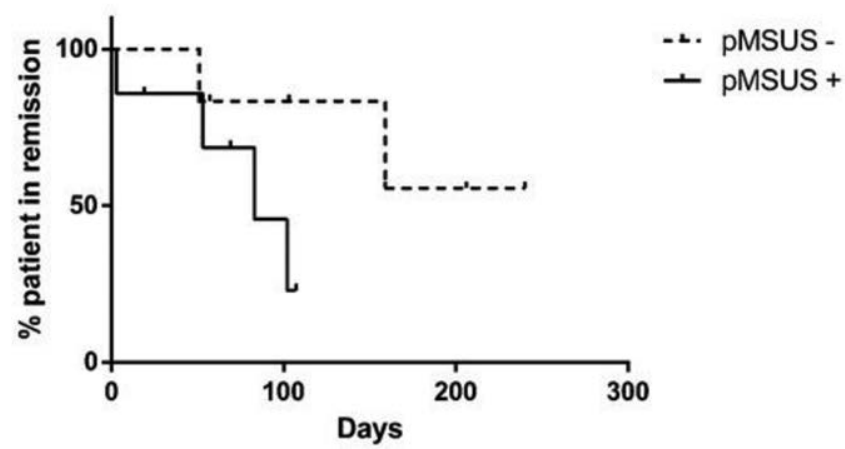

Conclusion: $\cdot$ Sub-S are present in $50 \%$ of patients in clinical remission $>12$ months.

-Sub-S in older patients interest often feet small joints; these Sub-S may be of mechanical origin and are not associated with disease flare.

-Sub-S increase during MTX tapering.

Further patients must be enrolled to understand if Sub-S excluding feet small joints may predict disease flare.

References:

[1] Amendment of the OMERACT US definitions of joints' features in healthy children using the DOPPLER technique. Collado $\mathrm{P}$ et al, Pediatr Rheumatol Online J. 2018.

[2] Preliminary Definitions for the Sonographic Features of Synovitis in Children. Roth J, et al; Arthritis Care Res. 2017.

Disclosure of Interests: Francesco Licciardi: None declared, Marta Dellepiane: None declared, Carlotta Covizzi: None declared, Fabiana Figus: None declared, Irene Azzolin: None declared, Davide Montin Speakers bureau: Not relevant for the topic, Annamaria lagnocco Grant/research support from: Abbvie, MSD and Alfasigma, Consultant of: AbbVie, Abiogen, Alfasigma, Biogen, BMS, Celgene Eli-Lilly, Janssen, MSD, Novartis, Sanofi and Sanofi Genzyme, Speakers bureau: AbbVie, Alfasigma, BMS, Eli-Lilly, Janssen, MSD, Novartis, Sanofi DOI: 10.1136/annrheumdis-2020-eular.4151

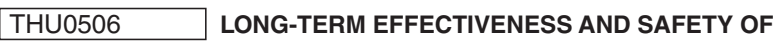 CANAKINUMAB AS A SECOND BIOLOGIC AFTER TOCILIZUMAB IN CHILDREN WITH EARLY AND LATE JIA WITH ACTIVE SYSTEMIC FEATURES}

E. Alexeeva $^{1,2}$, E. Krekhova ${ }^{2}$, T. Dvoryakovskaya ${ }^{1,2}, K^{2}$ Isaeva ${ }^{1}$,

A. Chomakhidze ${ }^{1}$, E. Chistyakova ${ }^{1,2}$, O. Lomakina ${ }^{1}$, R. Denisova ${ }^{1}$

A. Mamutova ${ }^{1}$, A. Fetisova ${ }^{1}$, M. Gautier ${ }^{1}$, D. Vankova ${ }^{1}$, M. Shingarova ${ }^{2}$,

A. Alshevskaya ${ }^{3}$, A. Moskalev ${ }^{3}$, I. Kriulin ${ }^{1,2} .{ }^{1}$ National Medical Research Center of Children's Health, Moscow, Russian Federation; ${ }^{2}$ Sechenov First Moscow

State Medical University (Sechenov University), Moscow, Russian Federation;

${ }^{3}$ Biostatistics and Clinical Trials Center, Novosibirsk, Russian Federation

Background: Canakinumab (CAN) is often used as second biologics in juvenile idiopathic arthritis with active systemic features (sJIA). However, there are little information about its long-term efficacy and safety.

Objectives: To evaluate the long-term effectiveness and safety of CAN as a second biologics after tocilizumab (TOC) in SJIA patients depending on the duration of the disease. 
Methods: Thirty-one patients were enrolled in this study: the group of early SJIA (with duration shorter than 2 years, 19 patients) and the group of late SJIA (with duration longer than 2 years, 12 patients). At the baseline, information was collected on the characteristics of the onset of the disease, previous therapy and its success. At each visit at least 1 time per year clinical and laboratory characteristics of SJIA severity were assessed. Response to therapy was assessed using the ACRPedi 30/50/70/90 criteria and the C.Wallace criteria for inactive disease (WID) and clinical remission.

Results: The most common reason for withdrawal of previous TOC was secondary ineffectiveness (22 cases, $71 \%$ ); in 6 cases (19.4\%) allergic reaction was observed; in two cases (6.5\%) primary non-effectiveness appeared; and in one case $(3.2 \%)$ there was marked infusion reaction.

At CAN initiation, sJIA activity was as follows: 15 (12: 23) for JADAS-71; 45 (36.5: 72) and 58 (45: 81) for physician's and patient's global assessment VAS; and 0.25 (0: 0.62) for the CHAQ disability index.

After 12-month treatment, 22 (71\%) patients reached WID: 21 on CAN therapy and 1 - after CAN withdrawal due to administrative reason and stable WID. ACR50/70/90 response was achieved by $84.2 \% / 84.2 \% / 64.7 \%$ patients in early arthritis group and in $83.3 \% / 75 \% / 75 \%$ patients in late arthritis group $(p=0.792)$.

However, $42.1 \%$ of patients with early SJIA achieved remission in the first 1.5 years without any further relapse during all the studied period and only $16.7 \%$ of patients with late arthritis $(p=0.239)$. In multivariable analysis, it was found that age of SJIA onset (OR $(2.5-97.5 \mathrm{Cl}) 0.353(0.13-0.72), p=0.015)$, number of joints with active arthritis at sJIA onset (2.308 (1.26-5.73), $p=0.025)$, and JADAS71 at SJIA onset $(0.664(0.44-0.88), p=0.016)$ were associated with successful treatment with rapid achievement of stable remission.

During the 76.7 patient-years follow-up period, 18 of $31(58.1 \%)$ patients were able to achieve a stable clinical remission and $27(87.1 \%)$ - WID. Two patients have achieved successfully drug-off remission. Serious adverse event (SAE) was reported in one (3.2\%) patient (enteritis).

Conclusion: Long-term canakinumab therapy proved to be effective and safe as a second biologics after tocilizumab for any duration of the disease. However, patients with early arthritis are more likely to quickly achieve stable remission without further relapse. Younger onset of SJIA with polyarthritis involvement and low disease activity are predictors of rapid and stable remission.

Disclosure of Interests: Ekaterina Alexeeva Grant/research support from: Roche, Pfizer, Centocor, Novartis, Speakers bureau: Roche, Novartis, Pfizer., Elizaveta Krekhova: None declared, Tatyana Dvoryakovskaya: None declared, Ksenia Isaeva: None declared, Aleksandra Chomakhidze: None declared, Evgeniya Chistyakova: None declared, Olga Lomakina: None declared, Rina Denisova: None declared, Anna Mamutova: None declared, Anna Fetisova: None declared, Marina Gautier: None declared, Dariya Vankova: None declared, Meyri Shingarova: None declared, Alina Alshevskaya: None declared, Andrey Moskalev: None declared, Ivan Kriulin: None declared

DOI: 10.1136/annrheumdis-2020-eular.5706

\section{THU0507 ASSOCIATION BETWEEN JUVENILE IDIOPATHIC ARTHRITIS AND AUTISM}

\section{R. Beesley ${ }^{1}{ }^{1}$ Juvenile Arthritis Research, Tonbridge, United Kingdom}

Background: Juvenile Idiopathic Arthritis (JIA) is a heterogenous group of autoimmune disorders characterised by chronic joint inflammation, diagnosed in around 1 in 1,000 children and young people (CYP) under the age of 16. Autistic Spectrum Condition (ASC) is a neurodevelopmental condition characterised by differences in social communication and sensory perception, as well as restricted interests and repetitive behaviours. Recent estimates from the Centers for Disease Control and Prevention (CDC) suggest that $1.68 \%$ of CYP are diagnosed with ASC, with males being more likely to be diagnosed (sex ratio of $4: 1$ ) [1]. The causes of both JIA and ASC are complex interactions between genetic and environmental factors. There appears to be some evidence that ASC may be associated with certain parental autoimmune conditions [2], although research into any association between JIA and ASC is sparse with the exception of a review of clinical database information [3].

Objectives: In this parent-led study, the association between JIA and ASC was explored in order to determine if children with JIA, or children who do not themselves have JIA but have at least one first-degree relative with JIA (FDR), are more likely to be diagnosed with ASC.

Methods: Parents of CYP with JIA were invited to complete an online survey, giving details of each member of their family including diagnosis status for JIA and ASC, and age of diagnoses. A total of 247 responses were collated, representing 558 CYP. Overall, 202 CYP were diagnosed with JIA from 197 families. The eldest child with JIA from each family was selected (total 197; 66 male and
131 female) and the rate of ASC was compared against the general population using Fisher's exact tests.

Results: Children with JIA themselves and FDR children were significantly more likely to be diagnosed with ASC.

\begin{tabular}{llll}
\hline Group & Odds Ratio $(95 \%$ Cl) & p-value & \\
\hline JIA children overall & $6.107(1.760,21.190)$ & 0.0020 & $\star \star$ \\
FDR children overall & $7.009(2.033,24.160)$ & 0.0006 & $\star \star \star$ \\
\hline
\end{tabular}

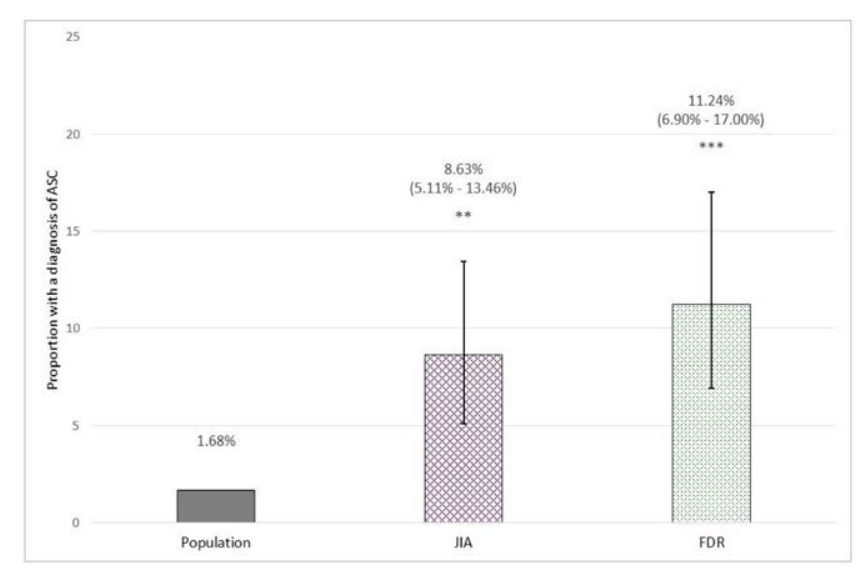

Figure 1. Proportion of children diagnosed with ASC in the general population (CDC estimates), JIA group and FDR group. Error bar indicates $95 \% \mathrm{Cl}$. Significance indicated compared to population.

Conclusion: Individuals with JIA and family members of individuals with JIA are more likely to be diagnosed with ASC. The results remained unchanged in a sensitivity analysis in which JIA children who had another sibling with JIA were excluded in order to minimise the risk that these results were affected by selecting the eldest child with JIA.

It is possible that we are underestimating the association between JIA and ASC in this study. The majority of children sampled were from the United Kingdom and Ireland; however, we chose to utilise the most recent CDC estimates for ASC prevalence, as the most recent estimates from the UK were from 2006 and longitudinal data suggests that ASC prevalence continues to increase, likely due to changes in diagnostic criteria and improved recognition of the condition. When using the UK prevalence estimates, JIA children and FDR children remain significantly more likely to be diagnosed with ASC than the general population as a whole.

Future research should focus on confirming these findings in larger, population-based samples.

\section{References:}

[1] Christensen DL, Braun KV, Baio J, et al. Prevalence and Characteristics of Autism Spectrum Disorder Among Children Aged 8 Years - Autism and Developmental Disabilities Monitoring Network, 11 Sites, United States, 2012. MMWR Surveill Summ (2018); 65 (No. SS-13):1-23.

[2] Hughes, H. K., Mills Ko, E., Rose, D. \& Ashwood, P. Immune Dysfunction and Autoimmunity as Pathological Mechanisms in Autism Spectrum Disorders. Frontiers in Cellular Neuroscience (2018); 12

[3] Haslam, K. P16 Is there an association between paediatric rheumatological disease and autism? Rheumatology 2019; 58

Disclosure of Interests: None declared

DOI: 10.1136/annrheumdis-2020-eular.876

\section{THU0508 \\ LARGE VESSEL VASCULITIS IN A COHORT OF CHILDREN WITH RESISTANT KAWASAKI DISEASE IN SINGAPORE}

$\underline{\text { L. Das }}^{1}$, J. H. T. Tan ${ }^{1}$, T. Arkachaisri ${ }^{1,2} .{ }^{1}$ KK Women's and Children's Hospital, Rheumatology \& Immunology, Singapore, Singapore; 'Duke-NUS Medical School, Singapore, Singapore

Background: Kawasaki Disease (KD) is one of the most common systemic vasculitidies in children today. IVIG is the mainstay of treatment, however, about $1 / 5$ of patients do not respond resulting in an increased risk of Coronary Artery Abnormalities (CAA) ${ }^{1}$. 